\title{
Probing the evolution of antiferromagnetism in multiferroics
}

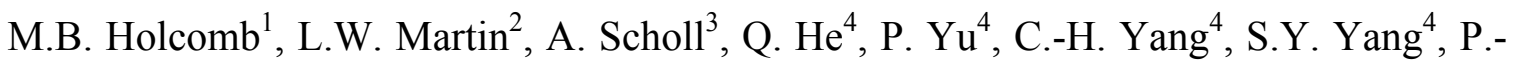

A. Glans ${ }^{3}$, M. Valvidares ${ }^{2}$, M. Huijben ${ }^{5}$, J.B. Kortright ${ }^{2}$, J. Guo ${ }^{3}$, Y.-H. Chu ${ }^{1,2,6}$, R. Ramesh $^{2,4}$

${ }^{1}$ Department of Physics, West Virginia University, Morgantown, WV 26506

${ }^{2}$ Materials Science Division, Lawrence Berkeley National Laboratory, Berkeley, CA 94720

${ }^{3}$ Advanced Light Source, Lawrence Berkeley National Laboratory, Berkeley, CA 94720

${ }^{4}$ Department of Physics and Department of Materials Science and Engineering, University of California, Berkeley, Berkeley, CA 94720

${ }^{5}$ Faculty of Science and Technology, MESA ${ }^{+}$Institute for Nanotechnology, University of Twente, Enschede, The Netherlands, $7500 \mathrm{AE}$

${ }^{6}$ Department of Materials Science and Engineering, National Chiao Tung University, HsinChu, Taiwan, 30010 
Motivated by the potential of coupled ferroelectric and magnetic properties in applications, this study aims to further elucidate the behavior of magnetic order in epitaxial films of the room temperature multiferroic $\mathrm{BiFeO}_{3}$ system. Using angle and temperature dependent dichroic measurements and spectromicroscopy, we have discovered that the antiferromagnetic order in the model multiferroic $\mathrm{BiFeO}_{3}$ evolves systematically as a function of thickness and strain. Lattice mismatch induced strain is found to break the easy-plane magnetic symmetry of the bulk and leads to an easy axis of magnetization which can be controlled. Additionally, the magnetic order is found to be diminished in ultrathin films representing the observation of systematic magnetic size effects in multiferroics over a wide range of film thicknesses. This understanding of the evolution of magnetic structure and the ability to manipulate the magnetism in this model multiferroic has significant implications for eventual utilization of such magnetoelectric materials in future applications.

Magnetoelectric multiferroics are the focus of much experimental and theoretical research $^{1-5}$ due to the potential to greatly impact the field of spin-based electronics (spintronics) including new forms of magnetic storage, logic, and memory. ${ }^{6-7}$ An ideal multiferroic would be a ferroelectric ferromagnet that exhibits coupling between these order parameters at room temperature. To date, such a material system has not emerged; instead, there is a strong focus on antiferromagnetic ferroelectrics, such as $\mathrm{BiFeO}_{3}$ (BFO), that can be coupled to ferromagnetic materials to achieve the desired coupling between electric fields and ferromagnetism at room temperature. ${ }^{6}$ 
To enable the design of such devices based on multiferroics, it is essential to understand the nature of the various order parameters especially in thin film samples. While conventional measurements, such as scanning force microscopy and quantitative polarization-voltage measurements can probe the evolution of the ferroelectric order parameter, probing the corresponding antiferromagnetic component in a multiferroic material is only achievable through optical probes or neutron scattering. ${ }^{1,8-9}$ Additionally, when the multiferroic is grown as epitaxial thin films for potential device applications, the antiferromagnetic state can evolve as a function of the constraints imposed by reduced dimensionality as well as from the strain imposed by the substrate. ${ }^{10}$ In this paper, we explore the evolution of antiferromagnetism, specifically the development of a preferred magnetic axis in thin films and the observation of size-effect induced diminished magnetism in ultra-thin films in model multiferroic BFO using state-of-the-art photoemission based spectromicroscopy at the Advanced Light Source, Lawrence Berkeley National Laboratory.

$\mathrm{BFO}$ is one of the most widely studied multiferroic materials over the last five years, largely because it is the only single-phase multiferroic material that is simultaneously both antiferromagnetic and ferroelectric at room temperature (Néel temperature $\left(\mathrm{T}_{\mathrm{N}}\right) \sim 643 \mathrm{~K}$ and ferroelectric Curie temperature $\left.\left(\mathrm{T}_{\mathrm{C}}\right) \sim 1103 \mathrm{~K}\right) .{ }^{11,12}$ This makes $\mathrm{BFO}$ an ideal candidate for use in room temperature applications. $\mathrm{BFO}$ is a rhombohedrally-distorted perovskite ferroelectric with large intrinsic polarization ${ }^{13}$ and eight possible polarization directions occurring along the pseudocubic $<111>$ body diagonals, one of which is shown in Figure 1(a). It is also known to be a G-type, canted antiferromagnet, ${ }^{13}$ so that the individual moments on each Fe-ion are aligned parallel 
within the pseudocubic $\{111\}$ and antiparallel between adjacent $\{111\}$. Additionally, recent density functional theory calculations for bulk BFO predict that the individual moments for each Fe-ion should lie along any one of six possible energeticallydegenerate magnetic axes of the $<1-10>$ - or $<112>$-type in an easy 111-type plane, which are perpendicular to the $<111>$ polarization direction, ${ }^{14}$ shown as the transparent red plane in Figure 1(a). Thus, one critical question concerning magnetism in multiferroics such as BFO that is of both fundamental and technological importance is how this order parameter develops with strain and size effects? In this paper, we demonstrate that such a systematic evolution of the state of magnetism can indeed be accomplished through a corresponding imposition of compressive (or tensile) strain on the bulk material. Our particular pathway is through an epitaxial constraint imposed by the substrate. We use angle-resolved, temperature-dependent photoemission electron spectromicroscopy (PEEM) to establish the fundamental origins of the magnetic state in BFO.

Single phase, epitaxial thin films of BFO were grown on a $50 \mathrm{~nm}$ thick conductive bottom electrode of $\mathrm{SrRuO}_{3}$ (SRO) on $\mathrm{SrTiO}_{3}$ (STO) (001) single crystal substrates via laser-molecular beam epitaxy and metalorganic chemical vapor deposition. Detailed x-ray diffraction studies coupled with transmission electron microscopy were used to establish the crystalline quality of the heterostructures; these films were found to be single phase and fully epitaxial. Throughout this article we refer to three classes of films: ultra-thin films with thickness $(\mathrm{t})<20 \mathrm{~nm}$, thin films that have $20 \mathrm{~nm}<\mathrm{t}<200$ $\mathrm{nm}$, and thick films that have $\mathrm{t} \sim 1 \mu \mathrm{m}$.

A combination of in-plane and out-of-plane piezoresponse force microscopy (PFM) allows 2D mapping of the ferroelectric polarization directions in a material. ${ }^{15}$ 
PFM is based on the detection of a bias-induced surface deformation in polar materials. Using a scanning probe setup, a tip is brought into contact with the surface of a material and the piezoelectric response of the surface is detected as the first harmonic component of the tip deflection during application of an $\mathrm{AC}$ bias to the tip. The phase of the electromechanical response of the surface yields information on the polarization direction below the tip. Details of the measurements are reported by Zavaliche, et al. ${ }^{15}$ A DIVecco Multimode AFM equipped with a Nanoscope IV controller has been used to simultaneously record the OOP and IP PR signals with the aim of reconstructing the polarization vector in each domain. Scans were performed at ambient temperatures and pressures using an $\mathrm{AC}$ bias of $6.3 \mathrm{kHz}$ applied to the conducting scanning probe tip. To image ferroelectric domains one can use the in-phase signals from a lock-in amplifier (Stanford Research Systems SR830). Final interpretation comes from recording images along various crystallographic directions.

The in-plane ferroelectric domain structure of one BFO thin film is shown in Figure 1(b); the long axis of the ferroelectric domains (or direction of the domain walls) lies along the $<100>$ of the underlying $\mathrm{SrTiO}_{3}(001)$ substrate or the pseudocubic- $<100>$ of BFO. The polarization direction of the various stripe-like domains lies along the $<111>$ and the in-plane projection of those polarization directions lies along the $<110>$. The arrows in Figure1(b) refer to the in-plane projection of the ferroelectric polarization directions (four variants) present in this model system. Such a model ferroelectric domain structure forms the reference frame for our photoemission measurements. 
Synchrotron-based x-ray absorption and photoemission spectromicroscopy have emerged as powerful tools that provide chemically specific insight into the nature of magnetic order in materials. ${ }^{16-18}$ Soft $\mathrm{x}$-ray absorption experiments were performed at the elliptically polarizing undulator beamline 11.0.1 at the Advanced Light Source of Lawrence Berkeley National Laboratory. Samples were measured in total electron yield (TEY), which consists primarily of inelastically scattered secondary electrons. The TEY sampling depth is approximately five nanometers. The photo-emitted electrons were extracted into an electron-optical imaging system by an electric field that is applied between the sample and the first electrode of the electron-optical system. Several electron-optical lenses are used to form a full field image of the emitted electrons onto a phosphor screen that converts electrons into visible light, which is detected by a CCD camera. Magnetic domains can be observed by dividing intensity maps where the absorption is different. For this study, linear dichroic images were obtained at $\pm 0.5 \mathrm{eV}$ from the Fe- $L_{3} \mathrm{~B}$ edge.

The $d$-shell properties largely responsible for the magnetism of transition metals and oxides are probed through $2 p$ to $3 d$ dipole transitions, i.e., $L_{3,2}$ absorption spectra, Fig.1(c). The $L$-edge spectra depend on anisotropies in the charge or the spin in the material, and thus are sensitive to the relative orientation of the x-ray polarization and AFM axes which are then imaged by PEEM. Recognizing that x-ray linear dichroism can arise from any anisotropic distribution of charge in a material, (as would be the case for both ferroelectric and antiferromagnetic order), we have decoupled the contributions from these sources unambiguously through temperature- and angle-dependent measurements. 
Figure 2(a) illustrates the $\mathrm{x}$-ray polarization geometry in which the XLD-PEEM images in Figures 2(b)-(e) were taken. The images in Figures 2(b)-(e) are from the corresponding area shown in Fig.1(b); for example, the "T" shape in Figures 1(b) and 2(b) are outlined as an aid to the reader. Images result from dividing intensity maps taken at the Fe- $L_{3}$ absorption edge using linearly polarized light as the angle of the plane of linear incident polarization $(\alpha)$ varies from $0^{\circ}$ (p polarization) to $90^{\circ}$ (s polarization). The outlined arrows in Figure 2 show the in-plane projection of the four ferroelectric directions. When the x-rays are s-polarized $\left(\alpha=90^{\circ}\right)$, only two contrast scales [labeled as light (1 and 3) and dark (2 and 4) in Figure 2(b)] are observed. We have chosen a geometry such that the x-ray polarization vector for an s-polarized incident beam is along [-110], which maximizes the difference in intensity between the domains. In this geometry, the propagation vector of the incident $\mathrm{x}$-rays is nearly parallel to [ $\left[\begin{array}{lll}1 & 1 & -1\end{array}\right]$, as shown in 2(a), due to the $\theta=30^{\circ}$ grazing angle from the sample surface. Figures 2(b)-(e) were obtained by changing the x-ray polarization angle $\alpha$ and keeping the grazing incidence angle $\theta$ fixed, which allowed us to gain insight into the magnetic structure by plotting the $\alpha$-angle dependence of the x-ray dichroic signal. Individual ferroelectric directions can be preferentially identified by rotating the polarization relative to the crystal as demonstrated in Figure 2(c). For example, in this figure, the technique highlights all [-1 $11-1]$-type ferroelectric domains (black arrow). The angle dependence reveals that at $\alpha=70^{\circ}$, three of the four $<111>$ type domains have similar contrast while the intensity from [-1 $11-1]$ domains is much higher. Further rotation of the polarization to $\alpha=40^{\circ}$ (Figure 2(d)) results in an image where all four ferroelectric variants can be distinguished due to their differences in contrast. Contrast between certain domain types 
can also vanish at other x-ray polarization angles-such as the [1-1-1] and [-11-1]-type domains in Figure 2(e) for $\alpha=0$.

Focusing on the image in Figure 2(c), where we have controlled the relative orientation of the incidence $\mathrm{x}$-ray polarization and the crystallographic orientation of BFO to preferentially observe one ferroelectric direction (in this case all ferroelectric domains of the [-11-1]-type), we gain insight into the nature of magnetism in BFO. We note again that all individual domains of a specific ferroelectric direction exhibit identical contrast. This indicates that the antiferromagnetic order in a given set of identical ferroelectric domains is the same. This automatically rules out the possibility of a magnetic structure such as that in a bulk sample of BFO in which there is a 111-type easy plane of magnetization perpendicular to the polarization direction. PEEM imaging in this geometry would result in multiple contrast levels for a given set of identical ferroelectric domains if the antiferromagnetic domains were larger then the PEEM resolution $(\sim 30$ $\mathrm{nm})$. The formation of antiferromagnetic domains with sizes smaller than $30 \mathrm{~nm}$ is highly unlikely, purely due to energetic considerations. ${ }^{16}$ Therefore, the data in Figure 2(c) is consistent with a magnetic structure in which an easy magnetic axis is formed in the $\{111\}$-magnetization plane of BFO similar to what is observed in strained $\mathrm{NiO}$ films. $^{10}$

Simple models of the x-ray polarization angle $(\alpha)$ dependence of the dichroic domain contrast (Figure 3) further support this conclusion. From a series of images (such as those in Fig. 2(b)-(e)), we can extract the $\alpha$ dependence of dichroic contrast (from both ferroelectric and magnetic contributions) for thin (Figure 3(a)) and thick (Figure 3(b)) films and compare this data to model calculations as a function of the $\mathrm{x}$-ray polarization 
angle $\alpha$ (Figure 3(a)). The angle dependence of the magnetic contribution to linear dichroism of an antiferromagnet has been modeled ${ }^{19}$ previously as

$$
\mathrm{I}=\left(3 \cos ^{2} \Theta_{\mathrm{M}}-1\right)<\mathrm{M}^{2}>_{\mathrm{T}}
$$

where $M$ is the magnetic moment at a temperature $T$ and $\Theta_{M}$ is angle between the AF axis and the incident $\mathrm{x}$-ray polarization vector axis. The ferroelectric contribution is also thought to exhibit a cosine-squared angular dependence ${ }^{15,20}$ on the angle $\Theta_{\mathrm{F}}$ between the ferroelectric polarization axis and the x-ray polarization axis. Therefore, we have modeled the dichroism from a multiferroic material with both magnetic and ferroelectric order when linearly polarized light is incident upon the sample at a given temperature as

$$
\mathrm{I}_{\mathrm{XLD}}=\mathrm{P} \cos ^{2} \Theta_{\mathrm{F}}+\mathrm{Q} \cos ^{2} \Theta_{\mathrm{M}}
$$

where $|\mathrm{P}|+|\mathrm{Q}|=1$. Constants $\mathrm{P}$ and $\mathrm{Q}$ can be either positive or negative, where $|\mathrm{P}|$ and $|\mathrm{Q}|$ are, respectively, the percentage contribution of the polarization and magnetic components to the dichroism at the given temperature. We have considered both positive and negative values for the constants $\mathrm{P}$ and $\mathrm{Q}$ in the current study. The dashed lines in Figure 3(a) correspond to the selected images in Figure 2 and the colors of the curves match the in-plane projection of the ferroelectric directions and colored arrows displayed in Figures 1(b) and 2. We have investigated a large number of possible magnetic structures encompassing many individual directions within the easy magnetization plane, combinations of these directions, and other possible directions not limited to that magnetization plane.

Models of the x-ray polarization angle dependence of the dichroic contrast for the two candidate magnetic structures previously discussed- a unique magnetic axis versus an easy magnetic plane-are shown in Figures 3(c) and (d), respectively. By comparing 
the experimentally measured dichroic contrast (Figures 3 (a) and (b)) with that in the calculated angle dependencies, a clear correlation emerges. For thin films, the data clearly indicates an easy magnetic axis; in contrast, for thick films, the data strongly suggests an easy magnetic plane. We note that data for only 2 variants are presented for the thick films (due to experimental constraints). Regardless, clear differences in the magnetic structure between thin and thick BFO films are observed, presenting the first evidence for the formation of an easy or preferred magnetic axis in epitaxially-strained thin films of BFO.

The angle-dependent data and model for thin films of BFO (Figure 3(a) and (c)) can only be achieved by two types of easy axes: [1-10] and [112] for a given [11-1]polarization direction. The image contrast for these two scenarios can look the same due to the fact that $\mathrm{P}$ and $\mathrm{Q}$ in Eq. 2 are variables. However, previous temperature-dependent measurements have shown that the percentage of the dichroism at room temperature originating from the ferroelectric polarization $(\mathrm{P})$ is approximately $60 \pm 10 \%{ }^{2}$ Therefore, we present the results of our modeling from this range, since it reasonably reflects the dependence of the observed data. In order to uniquely identify this axis, we have carried out temperature-dependent measurements.

The two possible types of easy axes, $<1-10>$ and $<112>$, should exhibit different temperature dependence of the intensity distribution when imaged, for instance, at $\alpha=0^{\circ}$ (Fig. 2(a)). For the case of a $<1-10>$ preferred axis, the magnetic axis for the domains with the largest contrast ( 2 and 4 at section e in Figure 3(a)) is perpendicular to the electric field vector of the incident x-rays. Thus, the difference between these two domains is dependent only on the contribution from ferroelectric polarization (P), which 
does not change appreciably over this temperature range. ${ }^{2}$ However, in the case of a $<112>$ preferred axis, the axis is not perpendicular to the electric field vector of the $\mathrm{x}$ rays, and therefore a definite temperature dependence arising from the magnetic contribution to the dichroism can be expected. Equation 2 can be adapted to variable temperature measurements by requiring both $\mathrm{P}$ and $\mathrm{Q}$ to be a function of temperature. Recognizing that the ferroelectric polarization in BFO does not change appreciably over the temperature range from room temperature to the Néel temperature $\left(\sim 370^{\circ} \mathrm{C}\right),{ }^{21}$ we focus on the temperature dependence of $\mathrm{Q}$, which should go to zero at the Néel temperature. ${ }^{17}$ From this, one can then estimate a $\sim 40 \%$ reduction in total intensity at the Néel temperature $\left(370^{\circ} \mathrm{C}\right)$ and, by interpolation, a $\sim 20 \%$ reduction in contrast should be achieved from room temperature to $200^{\circ} \mathrm{C}$.

Figure 4 illustrates the contrast between these domains $(2-$ light grey and $4-$ black) that has been repeatedly taken at room temperature (Figure $4(\mathrm{a})$ ) and at $200^{\circ} \mathrm{C}$ (Figure 4(b)). These images exhibit four shades-white, light grey, medium grey and black. Light grey and black correspond to the domains with the largest contrast (domains 2 and 4), whose temperature dependence determines which magnetic axis is present. The medium grey is achieved for both 1 and 3 domains. As illustrated by the several $2 / 4$ locations plotted in Figure 4(c), the contrast was found to decrease by $\sim 17 \%$ upon heating, which is in reasonable agreement with the $<112>$ easy axis scenario. Continued contrast reduction was observed at higher temperatures, but sample damage at these temperatures began to threaten sample integrity and therefore we have not included data beyond $200^{\circ} \mathrm{C}$. We have completed measurements on a blank BFO film and films capped with a $2 \mathrm{~nm}$ thick layer of SRO pre-heated for one hour at $200^{\circ} \mathrm{C}$ in an attempt to 
minimize the impact of any surface chemistry effects; however, the same trend as expected for the $<112>$ case was observed in all cases.

It is important to understand the driving force for this change in the magnetic structure in BFO. Strain-driven changes in magnetic structure have been observed previously in other magnetic oxide systems which exhibit a strong coupling among spin, charge, orbital and lattice degrees of freedom; for example ref. 22. In these materials, strain-induced changes have been interpreted by considering the variation of the electronic hopping amplitude due to the change of bond lengths and bond angles. ${ }^{22}$ Strain has been shown to affect the nature of antiferromagnetism in oxides such as $\mathrm{NiO}^{10}$. It has been suggested that an orthorhombic contraction (i.e., that which would result from an inplane compressive strain) along [100] favors a $<110>$ easy axis; whereas a monoclinic expansion (i.e., that which would result from an in-plane tensile strain) along [110] favors a $<112>$ easy axis. ${ }^{23}$ The lattice mismatch between BFO and STO (1.54\%) imparts a compressive in-plane strain to the BFO film that is progressively relaxed as the film thickness is increased. ${ }^{24}$ Using x-ray reciprocal space mapping (RSM) of the 203 BFO diffraction peak as shown in Figure 5(a)-(b) we have studied the strain state in such films as a function of film thickness. The in-plane (a) and out-of-plane (c) lattice parameters as well as the monoclinic tilt angle $(\beta)$ are shown in the table along with a schematic describing the nature of the structural distortions (Figures 5(c) and (d)). In the case of a $200 \mathrm{~nm}$ thick BFO/SRO/STO (001) film, the in-plane lattice parameters are matched with those of the substrate and the unit cell is under compressive strain (c/a-1 $+3.9 \%)$ [Figure 5(a)]. As the film thickness is increased to $1 \mu \mathrm{m}$, the lattice parameters gradually approach the bulk BFO value and the pseudo-tetragonality (c/a-1) becomes smaller 
$(+0.53 \%)$ as measured from RSM (Figure $5(b)) . \beta$ is derived from the peak splitting of the 203-peak and is calculated to be $\sim 0.74^{\circ}$, close to the value measured in bulk BFO. The in-plane compressive strain lifts the degeneracy of magnetization in the 111-plane, as is also observed in the case of $\mathrm{NiO}$ thin films. ${ }^{10}$ This preferred axis can also be understood in terms of the magnetostriction in BFO. The magnetostriction constant of $\mathrm{BFO}$ is positive ${ }^{25}$ meaning that the lattice constants expand along an applied magnetic field. Substrate-induced compressive strain is effectively the opposite of this effect and thus results in a preference of a magnetization direction that has the largest out-of-plane component while remaining in the (11-1), i.e, the $<112>$. Similarly, preliminary findings suggest that $\mathrm{BFO}$ films under tensile strain (for example, by epitaxial growth on $\mathrm{Si}$ ) demonstrate the opposite axis preference within the (11-1), i.e., the in-plane $<1-10>$. This demonstrates that one can control the nature the magnetic axis in such multiferroic thin films and in turn use this ability to better engineer a new generation of devices that utilize electric field driven changes in magnetic order.

The evolution of both the ferroelectric and antiferromagnetic order parameters with the critical length scale, i.e., the film thickness, is the next important question. Sizeeffects in magnetic ${ }^{26-27}$ and ferroelectric ${ }^{24,28}$ materials have been extensively studied over the years and these studies have demonstrated that there is indeed a minimum thickness required to stabilize the order parameters needed for device functionality. Few studies, however, of size effects in multiferroics have been reported. Prior studies on size effects on ferroelectricity in ultra-thin BFO films, ${ }^{24,29}$ have demonstrated that ferroelectricity is present down to a film thickness of $\sim 2 \mathrm{~nm}$, but no study has yet examined the evolution of magnetic order in ultra-thin films. Figure 6 shows the XMLD asymmetry for BFO 
films ranging in thickness from 50 to $2 \mathrm{~nm}$. The XMLD spectroscopic measurements were completed by comparing spectra taken at $\theta=70^{\circ}$ to $\theta=33^{\circ}\left(\alpha=90^{\circ}\right)$ at beamline 7.0.1 at the ALS. There is a progressive decrease in magnitude of the asymmetry at thicknesses below $\sim 15-20 \mathrm{~nm}$, which corresponds very closely to the onset of similar size effects in antiferromagnetic $\mathrm{CoO}{ }^{26}$ Similar to the reduction of critical temperature observed in superconducting or ferroelectric thin films, antiferromagnets exhibit a loss in magnetic order when the film thickness approaches the material's correlation length. In turn, this diminished magnetic order in these ultra-thin BFO films deleteriously affects the interactions of the BFO layer with a ferromagnet via exchange coupling at an interface ${ }^{30}$. Data in Fig.6 shows the magnitude of the exchange bias field for a $5 \mathrm{~nm}$ thick ferromagnetic $\mathrm{La}_{0.7} \mathrm{Sr}_{0.3} \mathrm{MnO}_{3}$ layer for various thicknesses of $\mathrm{BFO}$. The magnitude of exchange bias, i.e., the anisotropic shift of the magnetic hysteresis loop of the ferromagnet, falls off concurrently with the diminished XMLD asymmetry (Fig. 6). Such a reduction in exchange bias as a result of diminished antiferromagnetic order has been observed in many classic exchange bias heterostructures. ${ }^{31}$ Although this is not the primary focus of this paper, the fundamental mechanisms by which both the order parameters begin to be suppressed with dimensions (i.e., film thickness) is an area that merits further detailed studies. From an applications perspective, it is clear that the evolution of magnetic order in thin films of multiferroics is a critical subject to study if researchers hope to develop a new generation of devices that utilizes these exciting materials.

In conclusion, the evolution of magnetism in multiferroic BFO is clearly a complex and intriguing subject. Through a careful experimental and theoretical study of 
PEEM images and the underlying structure of BFO (001), we have determined that epitaxially strained thin films do not show a degenerate magnetic plane as predicted for bulk, but instead exhibit the formation of a preferred magnetic axis depending on the nature of strain $(<112>$ or $<1-10>$ for compressive and tensile strain, respectively). Additionally, we have observed a classic size effect behavior for the antiferromagnetism in BFO that begins in films thinner than $\sim 20 \mathrm{~nm}$. These observations will enable a deeper understanding of the magnetic exchange interactions at an interface between such epitaxial BFO films and a ferromagnet and aid in the design of next generation devices. ${ }^{32}$ Finally, we believe this x-ray dichroism based spectromicroscopy technique can be generically applied to other multiferroic systems where magnetic behavior has been difficult to determine due to the complexity of multiple order parameters and thus represents a powerful tool in achieving unprecedented understanding of order in these exciting materials.

This work has been supported by the Director, Office of Basic Energy Sciences, Materials Science Division of the U.S. Department of Energy under Contract No. DEAC02-05CH11231. C.H.Y was supported by a Korea Research Foundation Grant (MOEHRD) (KRF- 2006-214-C00020). Y.H.C. would like to acknowledge the support of the National Science Council, R.O.C., under contract NSC 97-3114-M-009-001. MBH also acknowledges the support of the SRC-NRI for funding support. 
*Corresponding author; mikel.holcomb@mail.wvu.edu

${ }^{1}$ M. Fiebig, J. Phys. D: Appl. Phys 38, R123 (2005).

${ }^{2}$ W. Eerenstein, N. D. Mathur, and J. F. Scott, Nature 442, 759 (2006).

${ }^{3}$ S.-W. Cheong and M. Mostovoy, Nature Mat. 6, 13 (2007).

${ }^{4}$ R. Ramesh and N. A. Spaldin, Nature Mat. 6, 21 (2007).

${ }^{5}$ H. Bea, M. Gajek, M. Bibes, and A. Barthelemy, J. Phys. C 20, 434221 (2008).

${ }^{6}$ C. Binek and B. Doudin, J. Phys. C 17, L39 (2005).

${ }^{7}$ Science Magazine features Science Watch on Multiferroics: Breakthrough of the Year 2007. http://www.sciencemag.org/sciext/btoy2007/atw_links.html.

${ }^{8}$ A. Pimenov, A. A. Mukhin, V. Yu. Ivanov, V. D. Travkin, A. M. Balbashov, and A. Loidl, Nature Physics 2, 97 (2006).

${ }^{9}$ D. Lebeugle, D. Colson, A. Forget, M. Viret, A. M. Bataille, and A. Gukasov, Phys. Rev. Lett. 100, 227602 (2008).

${ }^{10}$ H. Ohldag, A. Scholl, F. Nolting, S. Anders, F. U. Hillebrecht, and J. Stohr, Phys. Rev. Lett. 86, 2878 (2001).

${ }^{11}$ C. Michael, J.-M. Moreau, G. D. Acheubach, and W. J. James, Solid State Commun. 7, 701 (1969).

${ }^{12}$ P. Fischer, M. Polomska, I. Sosnowska, and M. Szymanski, J. Phys. C 13, 1931 (1980).

${ }^{13}$ S. V. Kiselev, R. P. Ozerov, and G. S. Zhdanov, Soviet Phys.-Doklady 7, 742 (1963).

${ }^{14}$ C. Ederer and N. A. Spaldin, Phys. Rev. B 71, 060401 (2005).

${ }^{15}$ F. Zavaliche, S. Y. Yang, T. Zhao, Y. H. Chu, M. P. Cruz, C. B. Eom, and R. Ramesh, Phase Trans. 79, 991 (2006). 
${ }^{16}$ J. Stohr and H. C. Siegmann, Magnetism From Fundamentals to Nanoscale Dynamics (Springer, New York, 2006).

${ }^{17}$ A. Scholl, H. Ohldag, F. Nolting, J. Stohr, and H. A. Padmore, Rev. Sci. Instr. 73, 1362 (2002).

${ }^{18}$ G. Cressy, C. M. B. Henderson, G. van der Laan, Phys. Chem. Minerals 20, 111 (1993).

${ }^{19}$ J. Stohr, A. Scholl, T. J. Regan, S. Anders, J. Luning, M. R. Scheinfein, H. A. Padmore, and R L. White, Phys. Rev. Lett. 83, 1862 (1999).

${ }^{20}$ T. Tsuboi, Phys. Rev. B 39, 2842 (1989).

${ }^{21}$ T. Zhao, A. Scholl, F. Zavaliche, K. Lee, M. Barry, A. Doran, M. P. Cruz, Y. H. Chu, C. Ederer, N. A. Spaldin, R. R. Das, D. M. Kim, S. H. Baek, C. B. Eom, and R. Ramesh, Nature Mat. 5, 825 (2005).

${ }^{22}$ A. J. Millis, A. Migliori, and T. Darling, J. of Appl. Phys. 83, 1588 (1998).

${ }^{23}$ T. Yamada, J. Phys. Soc. Jpn. 21, 664 (1966).

${ }^{24} \mathrm{Chu}$ YH et al. (2007) Ferroelectric size effects in multiferroic BiFeO3 thin films. Appl. Phys. Lett. 90:252906.

${ }^{25}$ Zvezdin AK et al. (2006) Magnetoelectric interaction and magnetic field control of electric polarization in multiferroics. Jour. of Magnetism and Magnetic Materials 300 (1): 224-228.

${ }^{26}$ Ambrose T and Chien CL (1996) Finite-size effects and uncompensated magnetization in thin antiferromagnetic CoO layers. Phys. Rev. Lett. 76(10):1743-1746.

${ }^{27}$ Seo JW et al. (2008) Antiferromagnetic $\mathrm{LaFeO}_{3}$ thin films and their effect on exchange bias. J. Phys. Cond. Mat. 20: 264014. 
${ }^{28}$ Setter N et al. (2006) Ferroelectric thin films: Review of materials, properties, and applications. J. of Appl. Phys. 100(5):051606.

${ }^{29}$ Bea H et al, Jpn. J. Appl. Phys. 45, L187 (2006)

${ }^{30}$ Huijben $\mathrm{M}$ et al. (2009) Electrically controlled magnetization in an oxide multiferroic/ferromagnet heterostructure. Nature Materials, in review.

${ }^{31}$ Nogues J and Schuller I (1999) Exchange bias. Jour. of Magnetism and Magnetic Materials 192 (2): 203-232.

${ }^{32} \mathrm{Chu}$ YH et al. (2008) Electric-field control of local ferromagnetism using a magnetoelectric multiferroic. Nat. Mat 7 (6) 478-482.

\section{Figure Captions}

FIG. 1 (Color) Understanding order parameters in multiferroic BFO. (a) Schematic diagram of (001)-oriented BFO crystal structure with polarization along $<111>$ and predicted perpendicular easy magnetic plane for the bulk (shown in red). (b) PFM image of in-plane polarization projections with PFM and PEEM geometries (taken separately), showing incident x-rays 30 degrees from sample surface. (c) Absorption spectra of iron edge showing selected imaging energies.

FIG. 2 (Color) PEEM images of BFO at several angles of the electric vector of incident linear polarization $\alpha$. (a) Schematic illustrating the experimental geometries used to probe the angle dependent linear dichroism in BFO. The outlined arrows show the in-plane 
projection of the four ferroelectric directions. Images of domain structures taken at (b) $\alpha=90^{\circ}$, (c) $\alpha=70^{\circ}$, (d) $\alpha=40^{\circ}$, and (e) $\alpha=0^{\circ}$.

FIG. 3 (Color) Comparison between experimentally measured and modeled angle dependent XLD contrast. Experimentally measured dichroic contrast as the x-ray polarization is rotated for both thin (a) and thick (b) BFO films. Error for all data points is less than 3\% of the observed maximum contrast difference. Models of XLD contrast for two magnetic structures, a unique magnetic axis (c) and an easy magnetic plane (d), are shown for the same sample orientation as in Figure 2.

FIG. 4 (Color) Temperature dependent dichroism measurements of BFO. XLD images taken at (a) room temperature and (b) $200^{\circ} \mathrm{C}$. The labeled spots in (a) and (b) represent a selection of locations used to probe the temperature dependent change in dichoric contrast. (c) Temperature dependent changes in intensity for type 2 and 4 domains for both temperatures reveals that the difference between the contrast from type 2 and 4 domains reduces by $17 \%$ at $200^{\circ} \mathrm{C}$. This is expected for the presence of a preferred magnetic [112] axis.

FIG. 5 (Color) The crystal structure of thin and thick BFO films grown on SRO/STO(001). RSMs for thin (a) and thick (b) BFO grown on SRO/STO(001). (c) Schematic illustrating the nature of the crystal structure of the BFO film, where $a$ is the in-plane lattice parameter, $c$ is the out-of-plane lattice parameter, and $\beta$ is the monoclinic distortion angle. (d) Unit cell parameters, as determined by RSM, for both strained (thin) and relaxed (thick) films. 
FIG. 6 (Color) [in Red, left axis] The XMLD asymmetry as a function of thickness for BFO thin films. Below a BFO thickness of $20 \mathrm{~nm}$, a reduction in the antiferromagnetic order is observed. Asymmetry was measured by comparing spectra taken at $\theta=33^{\circ}$ and $\theta$ $=70^{\circ}\left(\alpha=90^{\circ}\right)$. [in Blue, right axis] The anisotropic shift of the magnetic hysteresis loop of the ferromagnet $\mathrm{La}_{0.7} \mathrm{Sr}_{0.3} \mathrm{MnO}_{3}$ on $\mathrm{BFO}$ falls off with $\mathrm{BFO}$ thickness, concurrently with the diminished XMLD asymmetry. The fit is a guide to the eye. 


\section{Figure 1}

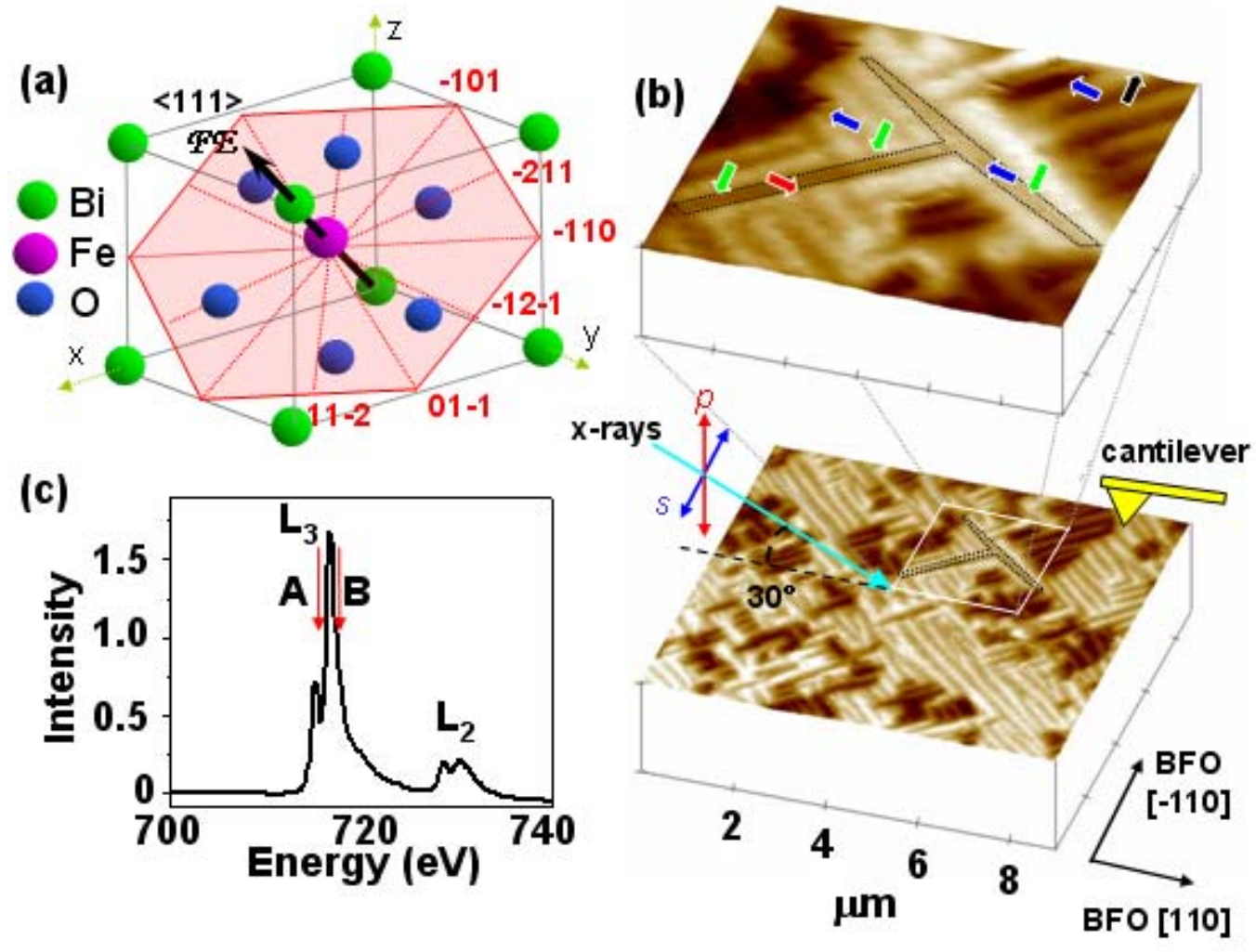




\section{Figure 2}
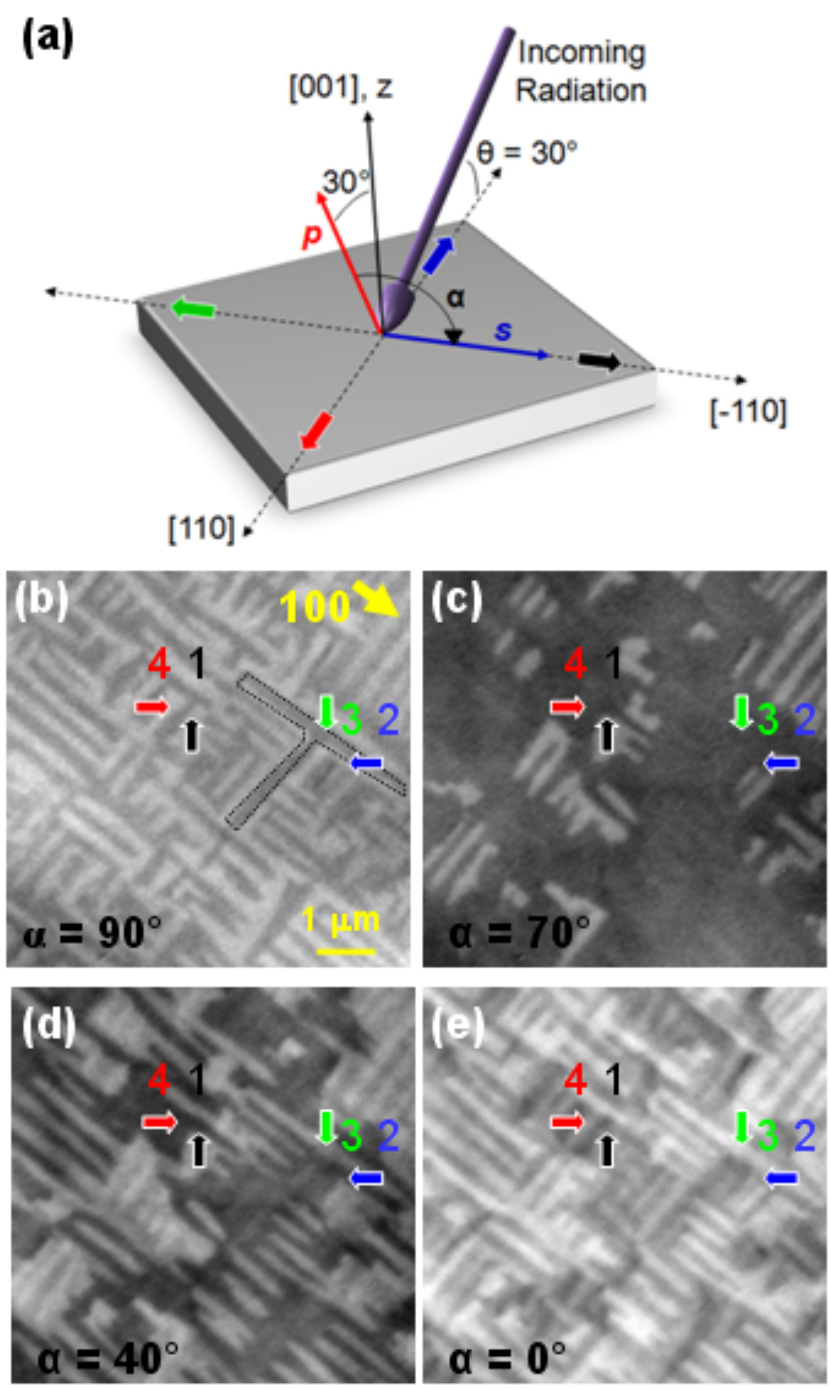
Figure 3
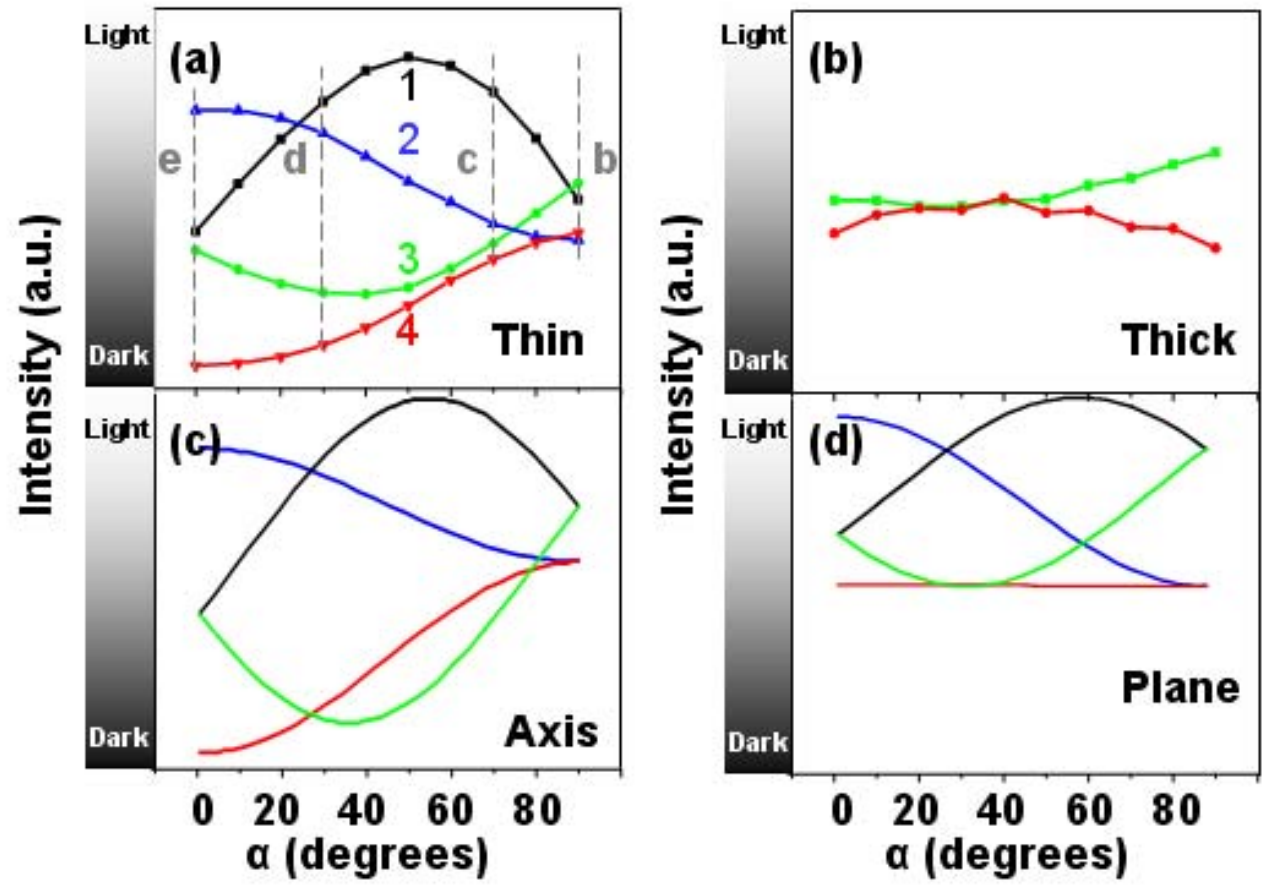


\section{Figure 4}

(a)

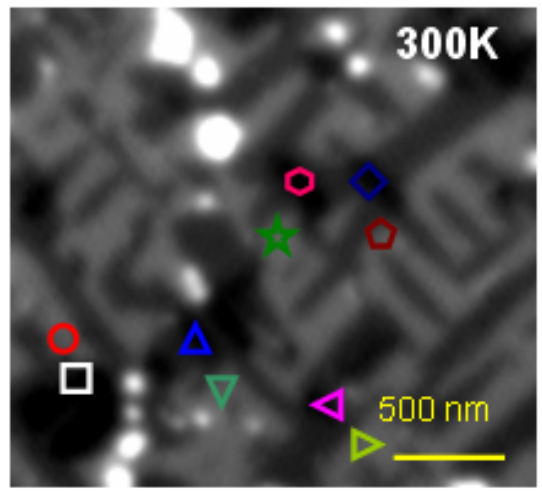

(b)

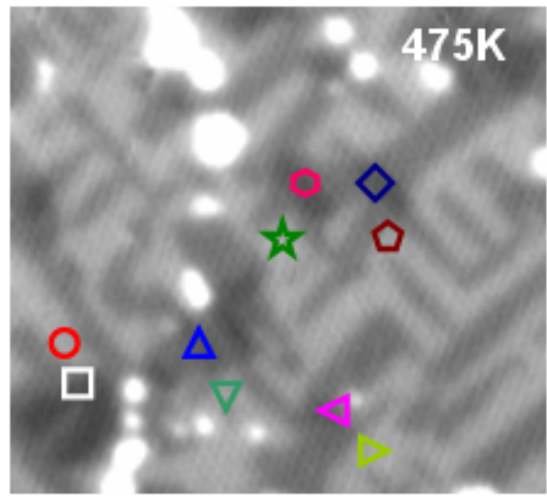

(c) 3900

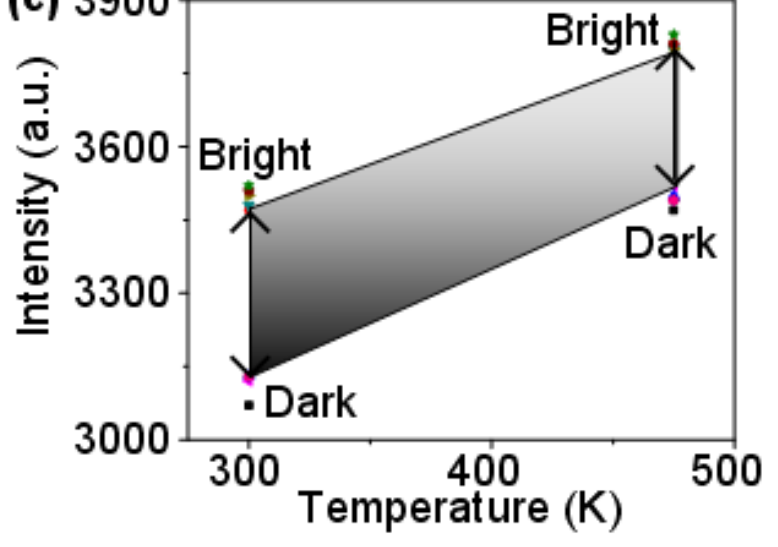


Figure 5

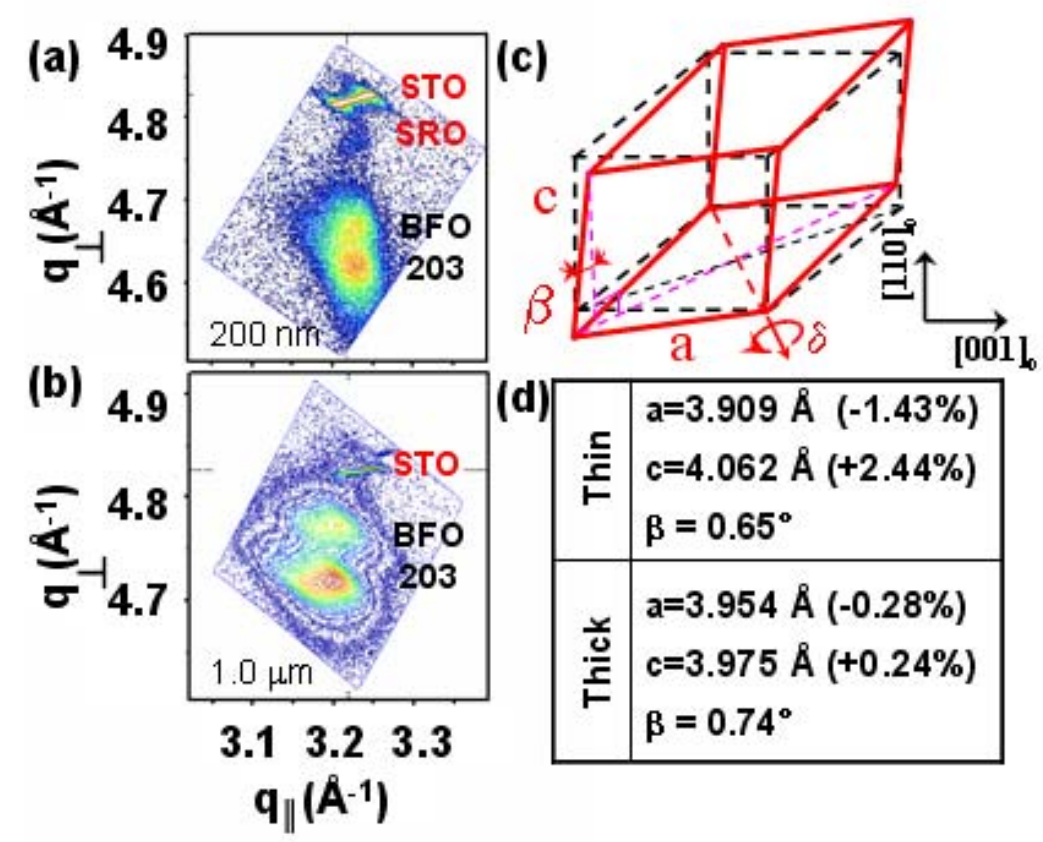


Figure 6

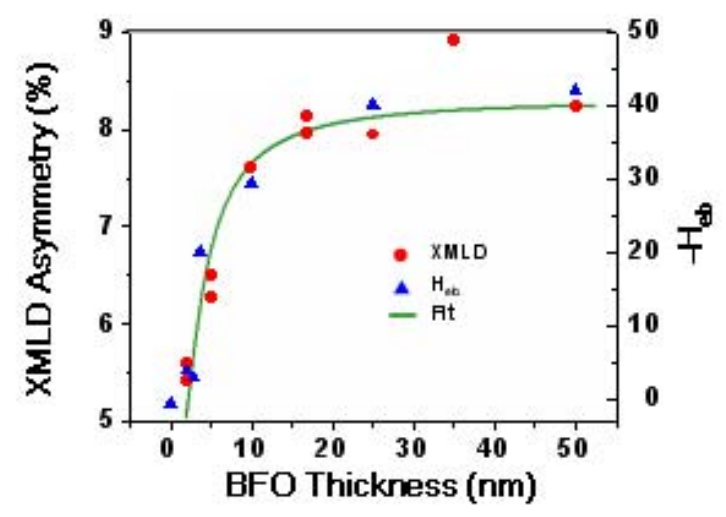

